\title{
The Value of Frontal Planar QRS-T Angle in Patients without Angiographically Apparent Atherosclerosis
}

\author{
Mutlu Gungor $^{\mathrm{a}}$ Murat Celik $^{\mathrm{b}}$ Emre Yalcinkayad Alper Tolga Polat ${ }^{\mathrm{a}}$ \\ Uygar Cagdas Yuksel $^{\mathrm{b}}$ Erkan Yildirim $^{\mathrm{b}}$ Serdar Firtina ${ }^{\mathrm{c}}$ Baris Bugan ${ }^{\mathrm{e}}$ \\ Ali Can Ozer ${ }^{a}$ \\ ${ }^{a}$ Department of Cardiology, Bayindir Hospital, and b Department of Cardiology, Gulhane Military Medical Academy,

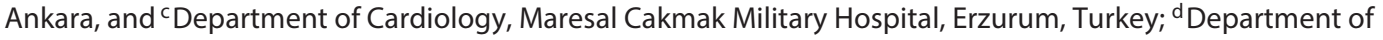 \\ Cardiology, Zurich University Hospital, Zurich, Switzerland; 'Department of Cardiology, Girne Military Hospital, \\ Girne, Cyprus
}

\section{Keywords}

Frontal planar QRS-T angle · Coronary angiography ·

Coronary artery diameter $\cdot$ Hypertension

\begin{abstract}
Objective: The present study was undertaken to investigate the prognostic value of the frontal planar QRS-T angle in patients without angiographically apparent coronary atherosclerosis. Subjects and Methods: Three hundred and seven patients with normal coronary arteries on coronary angiography were included. The absolute difference between the frontal QRS- and T-wave axes was defined as the frontal planar QRS-T angle, and patients were divided into 3 subgroups based on the frontal planar QRS-T angle (<45, 45-90, and $>90^{\circ}$ ). Demographic, clinical, laboratory, and angiographic data were compared between groups. Based on the regression analysis results, patients were recategorized into 4 groups according to their luminal calibers of left main coronary artery (LMCA) and history of hypertension (HT) (nonhypertensive LMCA $\leq 4.13 \mathrm{~mm}$, nonhypertensive LMCA >4.13 $\mathrm{mm}$, hypertensive LMCA $\leq 4.13 \mathrm{~mm}$, and hypertensive LMCA
\end{abstract}

\begin{tabular}{ll}
\hline KARGER & ( 2016 S. Karger AG, Basel \\
$\begin{array}{l}\text { E-Mail karger@karger.com } \\
\text { www.karger.com/mpp }\end{array}$ & $\begin{array}{l}\text { This is an Open Access article licensed under the terms of the } \\
\text { Creative Commons Attribution-NonCommercial 3.0 Un- } \\
\text { ported license (CC BY-NC) (www.karger.com/OA-license), } \\
\text { applicable to the online version of the article only. Distribu- } \\
\text { tion permitted for non-commercial purposes only. }\end{array}$
\end{tabular}

$>4.13 \mathrm{~mm}$ ). Results: The median value of the frontal planar QRS-T angle of all participants was $38^{\circ}$. Subjects with the widest frontal planar QRS-T angle were older ( $p=0.027)$, were hypertensive ( $p=0.001)$, and had higher corrected QT values $(p=0.001)$. Patients with the widest frontal planar QRS-T angle had larger LMCA and left anterior descending coronary artery diameters compared to subjects with a normal and borderline frontal QRS-T angle ( $p=0.004$ and $p=$ 0.028 , respectively). Corrected QT, HT, and LMCA diameter were found as independent predictors of the frontal planar QRS-T angle. Subjects with HT and a larger luminal caliber of LMCA had the widest frontal planar QRS-T angle. Conclusion: Patients with a history of HT and a larger luminal caliber of LMCA had the widest frontal planar QRS-T angle. Since HT-induced electrophysiological changes are still not well established and we observed that changes in the luminal caliber of coronary arteries are associated with an abnormal frontal QRS-T angle, the frontal QRS-T angle could serve as a marker of ventricular repolarization heterogeneity in hypertensive patients in addition to keeping track of arrhythmic events, even before overt disease.

(c) 2016 S. Karger AG, Basel

Emre Yalcinkaya, MD, FESC, ESC Training Fellow in Electrophysiology Department of Cardiology, Zurich University Hospital, University Heart Center Rämistrasse 100

CH-8091 Zurich (Switzerland)

E-Mail dremreyalcinkaya@gmail.com 


\section{Introduction}

Since vessel size has important therapeutic implications, coronary artery anatomy is a topic of interest because the measurement of the luminal diameter of the coronary arteries is an important indicator of early atherosclerosis [1]. Whereas smaller dimensions of the coronary arteries are a well-known independent risk factor for adverse cardiovascular events, larger dimensions are supposed to represent a variant of coronary atherosclerosis due to arterial remodeling [1]. The luminal caliber of the coronary arteries might be affected by age, sex, body surface area, autonomic function, anatomic distribution, branch length, and vessel tortuosity [2].

The QRS-T angle, defined as the angle between the mean QRS and $\mathrm{T}$ vectors, indicates the main orientation of electrical heart activity during ventricular depolarization and repolarization, and has recently become an area of research interest. A wider QRS-T angle reflects an abnormal arrangement of ventricular repolarization and has been considered as a strong and independent risk indicator for cardiac morbidity and mortality compared to other traditional cardiovascular risk factors and electrocardiographic (ECG) risk indicators such as the length of the QT interval [3]. The pathophysiological mechanisms underlying abnormalities of repolarization were mainly uncertain, but in a number of limited studies the predictive value and availability of the frontal planar QRS-T angle in various patient groups (hypertension [HT], coronary artery disease) have been investigated [4-6]. Also, there is no clear evidence about the effect of the luminal caliber of the coronary arteries on electrical activity of the heart.

We hypothesized that there might be a close relationship between coronary artery diameter and frontal QRS$\mathrm{T}$ angle. Therefore, the present study was undertaken to measure the luminal diameter of the coronary arteries in an adult population without angiographically apparent atherosclerosis, and to investigate whether or not there is a relationship between the diameters of the coronary arteries and the frontal planar QRS-T angle.

\section{Subjects and Methods}

\section{Patient Selection}

Patients in whom coronary angiography (CAG) was performed due to a clinical suspicion of coronary artery disease from March 2009 to May 2012 were analyzed. The exclusion criteria were coronary artery disease on CAG, a history of previous coronary artery bypass graft operation, atrial fibrillation, bundle branch block, ventricular or atrial premature depolarization, left ventricular systolic dysfunction, diabetes mellitus, uncontrolled HT (systolic blood pressure $>190 \mathrm{~mm} \mathrm{Hg}$ ), pericarditis, cardiomyopathy, presence of a pacemaker, and use of antiarrhythmic and vasodilator drugs. A total of 307 patients (males: 139, females: 168) with a normal coronary artery were included in the analysis. Demographic, clinical, laboratory, echocardiographic, and ECG data of the patients were obtained from the patients' medical records using the hospital's computerized database. The study was in compliance with the principles outlined in the Declaration of Helsinki, and the Institutional Committee approved the study protocol.

\section{Coronary Angiography}

Standard selective CAG using the Judkins technique with at least 4 views of the left coronary system and 2 views of the right coronary artery (RCA) were performed. The left main coronary artery (LMCA) was measured at its midpoint, the proximal left anterior descending coronary artery (LAD) was measured at its midpoint between its origin and the first septal or diagonal, the distal LAD was measured at its midpoint between the first and second diagonal, the left circumflex artery was measured at its midpoint between its origin and the first obtuse marginal, the proximal RCA was measured $20 \mathrm{~mm}$ from the ostium, and the measurement of the distal RCA was taken from the posterior descending artery. Measurements were obtained in 2 orthogonal views for each coronary artery. However, since the RCA is usually viewed only in 1 position, the second angiographic view could not be taken in most cases. The average of the 2 measurements was used for each coronary artery. Two separate investigators (M.G., A.C.O.) blinded to each other's results and patients' clinical data performed all measurements offline from the angiographic images on 2 separate occasions.

Surface ECG and Measurement of the Frontal QRS-T Angle

A resting 12-lead surface ECG with a paper speed of $25 \mathrm{~mm} / \mathrm{s}$ and a signal size of $10 \mathrm{~mm} / \mathrm{mV}$ was recorded in the morning period before CAG. The frontal plane $\mathrm{QRS}$ axis and $\mathrm{T}$-wave axis were included in the reports of the automated ECG machine. The absolute difference between the frontal QRS wave axis and T-wave axis was defined as frontal planar QRS-T angle. If such a difference exceeded $180^{\circ}$, the difference was calculated by subtracting from $180^{\circ}[7,8]$. After data collection, the patients were divided into 3 subgroups according to the frontal planar QRS-T angle: group 1 (normal) $=235$ patients with a frontal planar QRS-T angle of $0-45^{\circ}$, group 2 (borderline) $=41$ patients with a frontal planar QRS-T angle of $45-90^{\circ}$, and group 3 (abnormal) = the remaining 31 patients with a frontal planar QRS-T angle $>90^{\circ}$.

\section{Statistical Analysis}

The Statistical Package for Social Sciences (SPSS, Chicago, IL, USA) version 20 was used for all statistical analyses and calculations. The data were tested for normal distributions using the Kolmogorov-Smirnov test. Continuous variables such as the QRS-T angle are presented as means $\pm S D$ and the categorical variables as numbers and percentages. A $\chi^{2}$ test was used to compare categorical data. One-way analysis of variance (ANOVA) was used to determine significant differences among the means of the 3 groups. Spearman and Pearson correlation coefficients were used to perform univariate correlation between patients' data and coronary artery diameters. Multivariate linear regression analysis to deter- 
Table 1. Baseline characteristics of all participants and according to frontal planar QRS-T angles

\begin{tabular}{|c|c|c|c|c|c|}
\hline & $\begin{array}{l}\text { QRS-T angle } \\
0-45^{\circ}(n=235)\end{array}$ & $\begin{array}{l}\text { QRS-T angle } \\
45-90^{\circ}(n=41)\end{array}$ & $\begin{array}{l}\text { QRS-T angle } \\
>90^{\circ}(n=31)\end{array}$ & $\begin{array}{l}\text { All patients } \\
(n=307)\end{array}$ & $p$ value \\
\hline Age, years & $54.9 \pm 10.1$ & $56.7 \pm 11.6$ & $60.1 \pm 11.3$ & $55.7 \pm 10.5$ & 0.027 \\
\hline BMI & $30.43 \pm 5.43$ & $30.11 \pm 4.79$ & $31.30 \pm 6.81$ & $30.47 \pm 5.49$ & 0.640 \\
\hline Diabetes mellitus & $55(23.4)$ & $8(19.5)$ & $10(32.3)$ & $73(23.8)$ & 0.436 \\
\hline Hypertension & $109(46.4)$ & $29(70.7)$ & $22(71)$ & $160(52.1)$ & 0.001 \\
\hline LVEF, \% & $65 \pm 32$ & $61 \pm 9$ & $60 \pm 9$ & $64 \pm 28$ & 0.519 \\
\hline \multicolumn{6}{|l|}{ Biochemical data, mg/dL } \\
\hline LDL-C & $116.8 \pm 31.8$ & $124.3 \pm 35.7$ & $122.7 \pm 38.2$ & $118.4 \pm 33.1$ & 0.302 \\
\hline HDL-C & $46.8 \pm 13.1$ & $50.5 \pm 15.4$ & $49.2 \pm 13.8$ & $47.6 \pm 13.5$ & 0.225 \\
\hline Total-C & $194.9 \pm 36.7$ & $202.0 \pm 41.8$ & $197.2 \pm 49.3$ & $196.1 \pm 38.8$ & 0.553 \\
\hline Triglyceride & $164.1 \pm 99.0$ & $147.3 \pm 83.7$ & $141.0 \pm 58.9$ & $159.5 \pm 93.9$ & 0.294 \\
\hline Heart rate, bpm & $68 \pm 11$ & $70 \pm 12$ & $76 \pm 14$ & $69 \pm 12$ & 0.003 \\
\hline $\mathrm{P}$ axis & $43.6 \pm 25.8$ & $44.6 \pm 18.5$ & $50.1 \pm 48.2$ & $44.4 \pm 28.0$ & 0.515 \\
\hline QRS axis & $27.5 \pm 28.9$ & $18.8 \pm 43.5$ & $6.2 \pm 68.8$ & $24.2 \pm 37.3$ & 0.007 \\
\hline $\mathrm{T}$ axis & $31.5 \pm 27.0$ & $51.3 \pm 64.5$ & $101.2 \pm 70.8$ & $41.2 \pm 45.2$ & $<0.001$ \\
\hline Frontal QRS-T angle & $16.54 \pm 12.32$ & $62.97 \pm 10.75$ & $133.00 \pm 30.67$ & $34.50 \pm 39.52$ & $<0.001$ \\
\hline QTc & $408.7 \pm 27.1$ & $424.6 \pm 37.9$ & $422.7 \pm 38.4$ & $412.2 \pm 30.6$ & 0.001 \\
\hline
\end{tabular}

Values are given as means \pm SD or $n(\%)$. BMI, body mass index; LVEF, left ventricular ejection fraction; LDL-C, low-density lipoprotein cholesterol; HDL-C, high-density lipoprotein cholesterol; BUN, blood-urea nitrogen; QTc, corrected QT.

mine the independent variables likely to affect the frontal planar QRS-T angle was done. Based on the regression analysis, patients were recategorized into 4 groups according to the luminal calibers of the LMCA and history of HT: group A = nonhypertensive LMCA $\leq 4.13 \mathrm{~mm}$, group $\mathrm{B}=$ nonhypertensive $\mathrm{LMCA}>4.13 \mathrm{~mm}$, group $\mathrm{C}=$ hypertensive $\mathrm{LMCA} \leq 4.13 \mathrm{~mm}$, and group $\mathrm{D}=$ hypertensive LMCA $>4.13 \mathrm{~mm}$ was used to compare frontal planar QRS$\mathrm{T}$ angles between the groups with one-way ANOVA. Differences were considered significant at $p<0.05$.

\section{Results}

The mean age of all the patients was $55.7 \pm 10.5$ years. Of the 307 cases, 166 (54.1\%) had HT, 73 (23.8\%) had diabetes mellitus, and 88 (28.7\%) were smokers. The median value of the frontal planar QRS-T angle of all participants was $38^{\circ}$. Subjects with the widest frontal planar QRS-T angle were older $(60.1 \pm 11.3$ vs. $56.7 \pm 11.6$ and $54.9 \pm 10.1$ years $)(p=0.027)$ and hypertensive $(p=0.001)$. The corrected QT was higher in patients with the widest frontal planar QRS-T angle $(p=0.001)$. No association was found for other baseline demographic and clinical data. The baseline characteristics and biochemical data of all participants and of those with normal, borderline, and abnormal frontal planar QRS-T angles are given in Table 1 .

For all the patients, the mean luminal caliber of the LMCA was $4.18 \pm 0.61 \mathrm{~mm}$, the mean luminal calibers of the proximal and distal LAD were $3.32 \pm 0.49$ and $2.61 \pm$ $0.44 \mathrm{~mm}$, respectively, the mean luminal caliber of the left circumflex coronary artery was $3.11 \pm 1.08 \mathrm{~mm}$, and the mean luminal calibers of the proximal and distal RCA were $3.27 \pm 0.68$ and $2.48 \pm 0.67 \mathrm{~mm}$, respectively $(\mathrm{Ta}-$ ble 2). Patients with the widest frontal planar QRS-T angle had larger LMCA diameters and proximal LAD diameters compared to subjects with normal and borderline frontal QRS-T angle $(p=0.004$ and $p=0.028$, respectively). The corresponding parameters for normal, border line, and abnormal frontal QRS-T angles are listed in Table 2, whereas age, heart rate, corrected QT, HT, and LMCA diameter were significantly correlated with the frontal planar QRS-T angle in univariate correlation analysis; corrected QT, HT, and LMCA diameter were the independent variables that affected the frontal planar 
Table 2. Luminal calibers of all 3 coronary arteries according to the frontal planar QRS-T angles

\begin{tabular}{|c|c|c|c|c|c|}
\hline LMCA mean diameter, $\mathrm{mm}$ & $4.13 \pm 0.57$ & $4.20 \pm 0.61$ & $4.51 \pm 0.78$ & $4.18 \pm 0.61$ & 0.004 \\
\hline LAD proximal mean diameter, $\mathrm{mm}$ & $3.30 \pm 0.50$ & $3.26 \pm 0.48$ & $3.54 \pm 0.42$ & $3.32 \pm 0.49$ & 0.028 \\
\hline LAD distal mean diameter, $\mathrm{mm}$ & $2.59 \pm 0.44$ & $2.59 \pm 0.42$ & $2.79 \pm 0.42$ & $2.61 \pm 0.44$ & 0.051 \\
\hline CFX mean diameter, $\mathrm{mm}$ & $3.10 \pm 1.20$ & $3.04 \pm 0.54$ & $3.21 \pm 063$ & $3.11 \pm 1.08$ & 0.787 \\
\hline
\end{tabular}

LMCA, left main coronary artery; LAD, left anterior descending coronary artery; CFX, circumflex artery; RCA, right coronary artery.

Table 3. Effects of numerous variables on the frontal planar QRS-T angle in univariate correlation analysis and multivariate linear regression analysis

\begin{tabular}{|c|c|c|c|c|}
\hline & \multicolumn{2}{|c|}{ Univariate correlation analysis } & \multicolumn{2}{|l|}{ Multivariate regression analysis } \\
\hline & $r$ & $p$ value & $\begin{array}{l}\text { unstandardized coefficients B } \\
(95 \% \mathrm{CI})\end{array}$ & $p$ value \\
\hline Age & 0.170 & 0.003 & $0.285(-0.142$ to 0.711$)$ & 0.190 \\
\hline Heart rate & 0.142 & 0.013 & $0.272(-0.089$ to 0.634$)$ & 0.139 \\
\hline QTc & 0.209 & $<0.001$ & $0.224(0.085$ to 0.363$)$ & 0.002 \\
\hline Hypertension & 0.205 & $<0.001$ & $12.529(3.936$ to 21.121$)$ & 0.004 \\
\hline LMCA diameter & 0.229 & $<0.001$ & $11.421(4.356$ to 18.486$)$ & 0.002 \\
\hline LAD proximal diameter & 0.112 & 0.051 & $-4.303(-17.936$ to 9.331$)$ & 0.535 \\
\hline LAD distal diameter & 0.111 & 0.053 & $1.121(-15.240$ to 17.481$)$ & 0.893 \\
\hline CFX diameter & 0.038 & 0.508 & $-1.224(-5.409$ to 2.961$)$ & 0.565 \\
\hline RCA proximal diameter & 0.002 & 0.966 & $-3.385(-9.713$ to 2.943$)$ & 0.293 \\
\hline RCA distal diameter & -0.002 & 0.976 & $3.224(-9.031$ to 15.480$)$ & 0.605 \\
\hline
\end{tabular}

QTc, corrected QT; LMCA, left main coronary artery; LAD, left anterior descending coronary artery; CFX, circumflex artery; RCA, right coronary artery.

QRS-T angle in multivariate linear regression analysis (Table 3).

Based on the luminal calibers of the LMCA and history of HT, there was a statistically significant difference for frontal planar QRS-T angle among the 4 groups ( $p=$ 0.001 ) and this statistically significant difference was prominent between groups A and D (26.32 \pm 35.46 vs. $\left.47.30 \pm 48.94^{\circ}, p=0.002\right)$, and between groups B and D $\left(25.65 \pm 27.63\right.$ vs. $\left.47.30 \pm 48.94^{\circ}, p=0.004\right)$ (Fig. 1).

\section{Discussion}

The primary finding of this study was that there was an independent association between the frontal planar QRS-T angle and the luminal caliber of the LMCA and history of HT in subjects with normal coronary arteries on CAG. Subjects with a history of HT and a larger luminal caliber of the LMCA had the widest frontal planar QRS-T angle compared to other subgroups.

Despite many studies about the underlying pathophysiological mechanisms of ventricular repolarization abnormalities, we cannot find any literature data investigating the relationship between the luminal caliber of the coronary arteries and the frontal planar QRS-T angle in subjects with HT. With this article, a strong and independent relationship has now been fully defined between the coronary artery diameter and the frontal planar QRS-T angle. The findings of our study suggest that a higher, but still in the normal range, LMCA diameter might affect the electrical activity of the left ventricle in subjects with HT and without angiographically apparent atherosclerosis. 


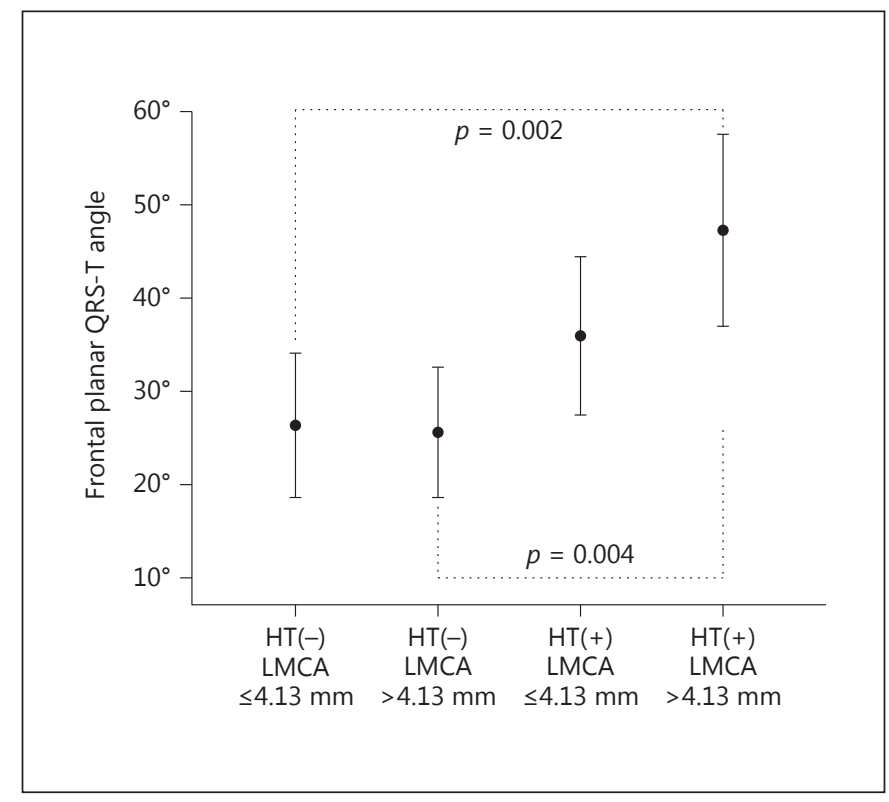

Fig. 1. Frontal planar QRS-T angles of subjects according to the presence of hypertension and luminal calibers of LMCA. LMCA, left main coronary artery.

Ventricular depolarization or repolarization abnormalities are important in arrhythmia provocation. The QRS-T angle is defined as the angle between the main electrical directions of ventricular depolarization and repolarization. It is easy to use and a more reproducible measurement than other conventional ECG parameters (especially QT dispersion), and shows fewer methodological limitations. Normally, as a result of the balanced regulation of electric activation, the orientation of the depolarization axis and repolarization axis is in a similar course, thereby resulting in a narrow QRS-T angle $\left(<45^{\circ}\right)$ [9]. However, a widened QRS-T angle reflects the increased ventricular repolarization heterogeneity and arrhythmic vulnerability [10].

In the present study, we used the frontal planar QRS$\mathrm{T}$ angle since most studies have shown that the frontal planar QRS-T angle is a proper clinical substitute for the spatial QRS-T angle for risk prediction [11] and the frontal planar axes of QRS and T are readily available in most ECG reports. Although the spatial QRS-T angle has a better prognostic value for cardiac risk prediction, the measurement is quite complex and needs dedicated software that is not commonly available and with which most clinicians are not accustomed to. In our study, we accepted the value of $>90^{\circ}$ for an abnormal frontal planar QRS-T angle, which is clearly outside the possible calculation errors since a frontal planar QRS-T angle $<45^{\circ}$ has been described as normal [12] and a QRS-T angle $>90^{\circ}$ is a powerful risk marker for cardiac mortality, independently of other clinical and ECG predictors $[2,13]$.

The QRS-T angle has recently been shown to be a strong and independent predictor of cardiac mortality for various patient groups such as coronary artery disease [5], heart failure [7], type 2 diabetes mellitus [14], elderly subjects [6], postmenopausal women [15], and Chagas disease [16]. Nonetheless, studies investigating the role of the QRS-T angle in HT are limited.

Elevated blood pressure is widely recognized to be an important cardiovascular risk factor for sudden death, of which cardiac arrhythmias are the main cause $[17,18]$. In general, high QT dispersion values have been reported in patients with systemic HT and left ventricular hypertrophy (LVH) [16]. However, the pathophysiological mechanisms underlying repolarization abnormalities in patients with HT were partially explained. Our findings suggest that measurement of the frontal planar QRS-T angle may help detect ventricular depolarization and repolarization abnormalities in patients with HT. In concordance with our study, Dilaveris et al. [19] showed that the QRS-T angle is a sensitive marker of the repolarization alterations in systemic HT, and Atsma et al. [20] showed that elevated blood pressure was directly related to cardiac repolarization disturbances, and the results were stronger for the QRS-T angle than for the $\mathrm{T}$ axis. The occurrence of arrhythmogenic electrophysiological changes in response to ventricular pressure or volume overload has been well documented [21]. HT causes $\mathrm{LVH}$ and thereby changes in shape and mass of the heart [22]. Myocardial hypertrophy alters the ionic channels during the early repolarization phase [23] and increases the collagen interstitial matrix [24] that may lead to ventricular repolarization alterations. As a result, LVH may generate or facilitate disturbances in ventricular depolarization and repolarization, subsequently favoring the propensity to cardiac arrhythmias and mortality [17]. Ishizawa et al. [25] showed that early changes in cardiac geometry and ventricular dimensions, even before overt LVH has developed, may result in changes of ventricular depolarization and repolarization, and may become visible on the ECG.

We found that subjects with a history of HT had larger LMCA compared to nonhypertensives ( $4.29 \pm 0.67$ vs. $4.06 \pm 0.51 \mathrm{~mm}, p=0.017$ ), but there was no statistically significant difference for the luminal diameters of other coronary arteries. In accordance with our study, Dodge et 
al. [26] found that the coronary luminal diameter was enlarged in patients with LVH and dilated cardiomyopathy. HT is associated with a change in the wall thickness-tolumen diameter ratio of the coronary artery and there is histological evidence of remodeling of coronary small arteries in HT [27-29]. This structural change is primarily due to eutrophic remodeling [30].

The present study's limitations included its retrospective nature based on a single center registry with a small number of patients, and the luminal caliber of the coronary arteries evaluated with CAG had some prognostic consequences. Cardiac magnetic resonance imaging could add more diagnostic information by visualizing not only the luminal caliber but also the arterial wall characteristics with high spatial and tissue contrast resolution; therefore, it is conceivable to use cardiac magnetic resonance imaging in a prospective design of such a study.

\section{Conclusion}

Patients with a history of HT and a larger luminal caliber of the LMCA had the widest frontal planar QRS-T angle. Further, the changes in the luminal caliber of the coronary arteries were associated with an abnormal frontal QRS-T angle; the frontal QRS-T angle could serve as a marker of ventricular repolarization heterogeneity in hypertensive patients in addition to keeping track of arrhythmic events, even before overt disease. However, further prospective studies are needed to clarify the underlying precise pathophysiology of ventricular repolarization abnormalities in hypertensive patients.

\section{Acknowledgement}

E.Y. was supported by a European Society of Cardiology Training Grant (T-2015-067).

\section{References}

1 Elezi S, Kastrati A, Neumann FJ, et al: Vessel size and long-term outcome after coronary stent placement. Circulation 1998;98:18751880.

2 Karakulak UN, Okutucu S, Şahiner L, et al: Assessment of cardiac autonomic nervous system involvement in systemic sclerosis via exercise heart rate recovery. Med Princ Pract 2015;24:17-22.

3 Voulgari C, Pagoni S, Tesfaye S, et al: The spatial QRS-T angle: implications in clinical practice. Curr Cardiol Rev 2013;9:197-210.

4 Dern PL, Pryor R, Walker SH, et al: Serial electrocardiographic changes in treated hypertensive patients with reference to voltage criteria, mean QRS vectors, and the QRS-T angle. Circulation 1967;36:823-829.

5 Palaniswamy C, Singh T, Aronow WS, et al: A planar QRS-T angle $>90$ degrees is associated with multivessel coronary artery disease in patients undergoing coronary angiography. Med Sci Monit 2009;15:MS31-MS34.

6 Walsh JA 3rd, Soliman EZ, Ilkhanoff L, et al: Prognostic value of frontal QRS-T angle in patients without clinical evidence of cardiovascular disease (from the Multi-Ethnic Study of Atherosclerosis). Am J Cardiol 2013;112: 1880-1884.

7 Raposeiras-Roubin S, Virgos-Lamela A, Bouzas-Cruz N, et al: Usefulness of the QRS-T angle to improve long-term risk stratification of patients with acute myocardial infarction and depressed left ventricular ejection fraction. Am J Cardiol 2014;113:1312-1319.
8 Rubulis A, Jensen J, Lundahl G, et al: T vector and loop characteristics in coronary artery disease and during acute ischemia. Heart Rhythm 2004;1:317-325.

9 Scherptong RW, Henkens IR, Man SC, et al: Normal limits of the spatial QRS-T angle and ventricular gradient in 12-lead electrocardiograms of young adults: dependence on sex and heart rate. J Electrocardiol 2008;41:648-655.

10 Brown RA, Schlegel TT: Diagnostic utility of the spatial versus individual planar QRS-T angles in cardiac disease detection. J Electrocardiol 2011;44:404-409.

11 Sur S, Han L, Tereshchenko LG: Comparison of sum absolute QRST integral, and temporal variability in depolarization and repolarization, measured by dynamic vectorcardiography approach, in healthy men and women. PLoS One 2013;8:e57175.

$12 \mathrm{Li} \mathrm{YH}$, Ren XJ, Han ZH, et al: Value of the frontal planar QRS-T angle on cardiac dysfunction in patients with old myocardial infarction. Int J Clin Exp Med 2013;6:688-692.

13 Aro AL, Huikuri HV, Tikkanen JT, et al: QRS$\mathrm{T}$ angle as a predictor of sudden cardiac death in a middle-aged general population. Europace 2012;14:872-876.

14 Voulgari C, Tentolouris N, Moyssakis I, et al: Spatial QRS-T angle: association with diabetes and left ventricular performance. Eur J Clin Invest 2006;36:608-613.
15 Rautaharju PM, Kooperberg C, Larson JC, et al: Electrocardiographic abnormalities that predict coronary heart disease events and mortality in postmenopausal women: the Women's Health Initiative. Circulation 2006; 113:473-480.

16 Zampa HB, Moreira DA, Ferreira Filho CA, et al: Value of the QRS-T angle in predicting the induction of ventricular tachyarrhythmias in patients with Chagas disease. Arq Bras Cardiol 2014;103:460-467.

17 Clarkson PB, Naas AA, McMahon A, et al: QT dispersion in essential hypertension. QJM 1995;88:327-332.

18 Belen E, Şahin İ, Güngör B, et al: Assessment of 25-hydroxyvitamin $\mathrm{D}$ levels in patients with resistant hypertension. Med Princ Pract 2016;25:25-30.

19 Dilaveris P, Gialafos E, Pantazis A, et al: The spatial QRS-T angle as a marker of ventricular repolarisation in hypertension. J Hum Hypertens 2001;15:63-70.

20 Atsma F, Bartelink ML, van der Schouw YT, et al: Elevated blood pressure and electrocardiographic frontal $\mathrm{T}$ axis and spatial QRS-T angle changes in postmenopausal women. J Electrocardiol 2008;41:360-364.

21 Eckardt L, Kirchhof P, Breithardt G, et al: Load-induced changes in repolarization: evidence from experimental and clinical data. Basic Res Cardiol 2001;96:369-380. 
22 Hegazy AM, Alkandary MH: Cardiac memory versus likelihood of ischemic heart disease in hypertensive patients with ventricular repolarization abnormalities after repetitive uniform ventricular extrasystoles. Med Princ Pract 2007;16:193-197.

23 Kleiman RB, Houser SR: Outward currents in normal and hypertrophied feline ventricular myocytes. Am J Physiol 1989;256:H1450H1461.

24 Pye MP, Cobbe SM: Mechanisms of ventricular arrhythmias in cardiac failure and hypertrophy. Cardiovasc Res 1992;26:740-750.
25 Ishizawa $\mathrm{K}$, Ishizawa $\mathrm{K}$, Motomura $\mathrm{M}$, et al: High reliability rates of spatial pattern analysis by vectorcardiogram in assessing the severity of eccentric left ventricular hypertrophy. Am Heart J 1976;91:50-57.

26 Dodge JT Jr, Brown BG, Bolson EL, et al: Lumen diameter of normal human coronary arteries. Influence of age, sex, anatomic variation, and left ventricular hypertrophy or dilation. Circulation 1992;86:232-246.

27 Tanaka M, Fujiwara H, Onodera T, et al: Quantitative analysis of narrowings of intramyocardial small arteries in normal hearts, hypertensive hearts, and hearts with hypertrophic cardiomyopathy. Circulation 1987; 75:1130-1139.
28 Schwartzkopff B, Motz W, Frenzel H, et al: Structural and functional alterations of the intramyocardial coronary arterioles in patients with arterial hypertension. Circulation 1993; 88:993-1003.

29 Chu G, Zhang G, Zhu M, et al: Acute one-stop cardiovascular magnetic resonance imaging for differential diagnosis in patients with acute coronary syndrome and unobstructed coronary arteries. Med Princ Pract 2015;24: 325-331.

30 Agilli M, Aydin I, Aydin FN: Atherosclerotic markers in obese and nonobese children and relationship with night time hypertension. Med Princ Pract 2014;23:584. 